\title{
Telomerase as a possible key to bypass the cost of reproduction effect
}

\author{
Radmila Capkova Frydrychova ${ }^{1}$ \\ ${ }^{1}$ Biology Centre Czech Academy of Sciences
}

January 4, 2022

\begin{abstract}
Telomerase activity and telomere restoration in certain somatic cells of human adults maintain the proliferative capacity of these cells and contribute to their regenerative potential, and telomerase activity and telomere length are commonly considered lifespan predictors. Eusocial insects provide excellent model systems for aging research based on their extraordinary caste-related lifespan differences that contradict the typical fecundity/lifespan trade-off. In agreement with the common presumption, telomerase activity is upregulated in the reproductive, long-lived individuals of eusocial insects such as queens and kings, proposing that telomerase activity acts as a key factor in their extended longevity. But, as documented by the presence of telomerase in somatic tissues of numerous invertebrate and vertebrate species, the connection between telomerase activity and the predicted lifespan is not clear. Here, I ask whether somatic telomerase activity in eusocial reproductives may serve its non-canonical function to protect its individuals against the exacerbated metabolic stress upon reproduction and be a reflection of a more common phenomenon among species. I propose a hypothesis that the presence of telomerase activity in somatic cells reflects a different reproduction strategy of the species.
\end{abstract}

\section{Introduction}

Social behavior is a set of interactions among individuals within a species and is displayed in a wide spectrum of forms, and eusociality represents the most intimate and complex degree of social organization. Eusociality is defined by the presence of distinct reproductive and non-reproductive castes, overlapping generations, and cooperative care for the brood within a colony (Engels 1990; Kocher and Paxton 2014). Reproductives (kings and queens) and non-reproductives (workers or soldiers) in the colonies are differentiated morphologically, physiologically, and behaviorally (Wilson 1971; Sherman et al. 1994; Engel 2012). Although the most well-characterized eusocial species are represented by termites and hymenopteran insect species (such as honeybees or ants), eusociality has been reported in several other insect, non-insect, or even vertebrate species (Sherman et al. 1994; Duffy et al. 2000).

Although taxonomically rare, eusocial insect species exhibit great ecological importance, as approximately $50 \%$ of the world insect biomass is represented by eusocial insect species (Wilson 1971; Kocher and Paxton 2014). Despite their abundance and renown, little is known regarding the ability of eusocial insects to offer an appealing opportunity for the scientific community to uncover mechanisms underlying how the social environment can alter the rate of organism aging. Due to their extraordinary caste-related lifespan differences, their plasticity in the rate of aging in the social context, and their contradiction of the usual fecundity/lifespan trade-off, eusocial insects provide an excellent model system for research on aging. This article proposes the possible role of telomerase and telomere biology in life-span regulation concerning the reproduction strategy of animal species.

\section{Eusociality contradicts a reversed reproduction-longevity trade-off}


The evolution theory according to natural selection attempts to explain how organisms maximize their fitness to achieve maximal genetic contribution within the future genetic pool. However, fitness maximization is limited by certain evolutionary constraints such as multiple life-history trade-offs. Based on the competitive allocation of limited resources, trade-offs act as negative correlations between the fitness components when an improvement of one component is associated with a decrement of the other (Fabian and Flatt 2014). One of the major trade-offs is the cost of reproduction, which is the phenomenon where the increased rate of reproduction reduces the longevity of the parent (Reznick 1985; Harshman and Zera 2006). However, the reversed reproduction-longevity trade-off is contradicted by eusocial insects, whose reproductive individuals, which are highly fecund compared to solitary insects, typically exhibit longer lifespans than do non-reproductives within their colonies or solitary insect individuals. Comparative analysis between the lifespan of eusocial reproductives and solitary insects has indicated that the evolution of eusociality is associated with a 100-fold increase in insect longevity (Jemielity et al. 2005). The reproductive individuals of many termite or ant species may live for many decades (Laurent and Genoud 1997; Keller 1998). For example, the mean average lifespans of honeybee queens is 5.6 years, while adults of solitary insect species exhibit lifespans of only 0.1 \pm 0.2 years (Keller and Genoud 1997; Keller 1998). The mechanisms underlying the extraordinary lifespan extension of eusocial reproductives are not fully understood.

\section{The aging explanation theories}

Aging is characterized by a series of cellular and tissue damage events that accumulate with age and result in impaired functioning of the organism that eventually leads to death. Although countless theories have been proposed as universal explanations for the aging process, none of these theories are entirely satisfactory. Instead, to explain the aging process these theories are applied in a complex way to provide a combinatorial explanation (Jin 2010).

Aging theories exist in two primary categories that include programmed and damage theories. The most established theory of aging is the mitochondrial free radical theory of aging, proposing that aging is a result of accumulating cellular damage caused by free radicals, and mitochondria act as the primary source of reactive oxygen species (ROS) (Harman 1956; Harman 1972). Generation of mitochondrial ROS such as hydroxyl radicals, hydrogen peroxide, and superoxide radicals primarily occurs during electron transport during oxidative phosphorylation. According to this theory, mitochondrial DNA (mtDNA) due to its proximity to ROS generation is considered the prime ROS target, and this targeting of mtDNA leads to the accumulation of ROS-induced damage in mitochondria and loss of functionality with age (Murphy 2009).

The programmed theory of aging proposes that aging is the result of a program that acts through gene expression where certain genes are switched on or off such as genes of the immune system or telomerase activity, both of which decline over time (Davidovic et al. 2010; Jin 2010; Fathi et al. 2019). Alternatively, aging is explained as a continuation of the program for developmental growth (acting as a quasi-program) that exerts a deleterious effect on adult individuals. Together with the antagonistic pleiotropy hypothesis, this theory is based on the assumption that evolution favors genes that increase fitness in early life despite the knowledge that they are harmful in later life (Blagosklonny 2010).

Regardless of any theory, aging is linked to multiple cellular processes that are interconnected and act in numerous cell organelles (Zheng et al. 2019). It has been demonstrated that the aging process is associated with changes in epigenomic information (Chittka and Chittka 2010; Lyko et al. 2010; Ben-Avraham et al. 2012; Herb et al. 2012; He et al. 2017) that affect the expression levels of aging-related genes (Kaiwen et al. 2018) and lead to alterations in numerous aging-related factors such as antioxidant protection (Kurz et al. 2004; Corona et al. 2005), nutrient-sensing pathways, energy homeostasis, and mitochondrial functioning (Ozawa 1997; Aamodt 2009; Blagosklonny 2010; Cui et al. 2012; Antikainen et al. 2017).

\section{Telomeres as a component of organismal aging}

Accumulating evidence exists indicating that stem cell function, regeneration, and organ maintenance, all of which largely contribute to the aging process, are connected to telomere biology. Telomeres are nucleoprotein structures located at chromosome ends that consist of short DNA repeats with well-defined sequence com- 
position and telomere-specific protein complexes. Through a multiprotein structure called a telomere cap, telomeres allow cells to distinguish natural chromosome ends from chromosome breaks, and the formation of telomere caps requires a satisfactory length of telomeric DNA (Blackburn 1991; Capkova Frydrychova et al. 2009; Mason et al. 2011). Disruption of telomere cap function that can occur either by loss of telomeric DNA or telomere-binding proteins activates the DNA damage response, and this response, in turn, induces a permanent proliferation arrest known as cell senescence (Greenberg 2005).

Due to the limitations of semiconservative DNA replication and the inability of conventional DNA polymerase to fully replicate the end of linear DNA strands, telomeres are gradually shortened with each round of cell division. This shortening can be circumvented by the extension of telomeric DNA via special telomere maintenance mechanisms, and the most common mechanism of telomere elongation involves telomerase activity. Telomerase is a specialized reverse transcriptase that uses its RNA template to repeatedly synthesize a short telomeric sequence onto the chromosome ends (Blackburn 2005; Mason et al. 2015). Telomerase activity is strictly controlled. In humans, the highest telomerase activity is observed during embryogenesis, and telomerase activity is downregulated in most somatic cells during later development. This suggests an important role for telomerase in fetal tissue differentiation and development (Wright et al. 1996; Ulaner and Giudice 1997). In adult humans, the majority of somatic cell types are telomerase-negative, and telomerase activity is primarily present in germ, stem, and cancer cells. In contrast to germ and cancer cells, the level of telomerase in most stem cells of human adults is low and insufficient to prevent replicative senescence (Hiyama and Hiyama 2007; Choudhary et al. 2012). Telomerase in adult humans is also upregulated in cells with high reproducible activity such as hematopoietic progenitor cells, endometrial and intestinal cells, activated lymphocytes, or keratinocytes (Wright et al. 1996; Razgonova et al. 2020). In contrast to other cell types, embryonic stem cells and cancer stem cells are, due to their high telomerase activity, considered immortal having the capacity of indefinite self-renewal and proliferation (Hiyama and Hiyama 2007).

The absence of or low activity of telomerase in somatic cells results in significant telomere shortening throughout the lifespan, and this limits the replicative capacity of the cells and acts not only as a major determinant of organismal development but also aging and age-related diseases (Ulaner and Giudice 1997; Jiang et al. 2007; Razgonova et al. 2020). Also, as documented in zebra finches, there is a positive correlation between telomere length early in life and the length of realized lifespan, thus indicating that the length of telomeres may act even as a predictor of lifespan predisposition (Heidinger et al. 2011).

\section{Telomerase is upregulated in the long-lived eusocial reproductives}

The common presumption that telomerase activity is a marker of aging and advancing organismal development even in insects is supported by observations in hemimetabolous insects such as cockroaches and termites (Korandová et al. 2014; Koubová and Čapková Frydrychová 2021). Hemimetabolous insects exhibit incomplete metamorphosis, where during development the insects lack larval and pupal stages and instead undergo several nymphal stages before their final molt into adults. Recent phylogenetic studies indicate that termites evolved from cockroaches, and along with cockroaches, they form the order Blattodea. But, in contrast to cockroaches, termites are eusocial insects. Both cockroaches and termites exhibit upregulated telomerase activity in young instars, and this activity gradually declines in later development. However, there were two exceptions for the decline: the germline cells in both insects and somatic tissues in the long-lived reproductives, exhibiting high telomerase activity throughout the duration of their lives (Korandová et al. 2014; Koubová and Čapková Frydrychová 2021). A similar finding, which contradicts the common scheme of telomerase decline in adult somatic tissues, was obtained in the long-lived honeybee and ant queens (Korandová and Frydrychová 2016; our unpublished data), representing holometabolous insects (adult growth in holometabolous insects is largely determined and ended by metamorphosis). These observations suggest that the upregulation of telomerase activity may be a key factor in the caste differentiation process in eusocial insects and in the extended longevity of their reproductive individuals.

It is well established that if telomerase is required for the maintenance of telomeres, it must be active during the DNA replication stage (S-phase), and while the highest levels of telomerase activity are found in the S-phase, telomerase activity is virtually absent in the G2/M or G0 phases (Zhu et al. 1996; Holt et al. 1997). 
Surprisingly, no DNA synthesis was detected in any of the telomerase-positive somatic tissues of eusocial reproductive insects (Koubová and Čapková Frydrychová 2021), and no differences were observed in telomere length between the long-lived and short-lived castes (Jemielity et al. 2005; Korandová and Frydrychová 2016; Koubová et al. 2021a).

To explain the role of telomerase in the caste system of eusocial insects and to identify its possible engagement in the disparity between fertility and life expectancy, research was further conducted examining the bumblebee Bombus terrestris . Bumblebees are members of the group of insects possessing a primitive social organization, and there are significant differences in the life expectancy of their female castes. Workers of the bumblebee species $B$. terrestris typically live for 2-3 months; however, the queens can live up to one year. Nevertheless, the lifespan comparison is not unbiased, as the bumblebee queens spend the majority of their lives (approximately 6-9 months) in a diapause in which most biological processes take place at only low-cost levels. Thus, the bumblebee queens cannot provide an example of a full-bodied extension of life expectancy, or at least they cannot provide it in the way that exists in advanced eusocial species. In contrast to eusocial reproductive individuals, the only adult somatic tissue of $B$. terrestris showing upregulated telomerase was the fat body of very young and pre-diapause queens (Koubová et al. 2019). Additionally, telomerase activity was co-localized with the DNA endoreduplication cycles that were followed by a massive increase in fat body mass and nutrient content, which suggests that the upregulation of telomerase activity in the fat body mass in B. terrestris is tightly linked to the ability of queens to survive upcoming diapause (Koubová et al. 2019). A similar observation was obtained in honeybee workers, where telomerase activity, DNA synthesis, and nutrient content were reinforced in the fat body cells of winter-generation workers (Koubová et al. 2021b).

Collectively, these findings suggest that the caste-related differences in telomerase activity in eusocial insects such as honeybees or termites are not linked to telomere maintenance mechanisms, and instead, they are associated with some non-canonical telomerase roles without the typical telomerase catalytic activity that directly serves to elongate telomeres.

\section{Non-canonical functions of telomerase in oxidative defense}

An increasing body of evidence indicates that non-canonical telomerase functions participate in a variety of biological pathways related to DNA repair and stress resistance. In addition to the regulation of cell growth and the cell cycle, the induction of apoptosis, or changes in global patterns of gene expression or chromatin status, telomerase and telomeres are involved in protecting cells against oxidative stress (Saretzki 2009; Ségal-Bendirdjian et al. 2019; Zheng et al. 2019). The impact of telomere biology in regard to cell functions under oxidative stress is documented by the crosstalk between telomeres/telomerase and mitochondria. It was determined that mitochondrial dysfunctions accelerate telomere shortening, thus indicating that mitochondrial ROS may act as a determinant of telomere-dependent senescence (Liu et al. 2002; Passos et al. 2007) and that telomere shortage and dysfunction can lead to the reprogramming of mitochondrial biosynthesis and alterations in mitochondrial functioning (Guo et al. 2011; Sahin et al. 2011). Additionally, it has been reported that in response to oxidative stress induced by hyperoxia, $80-90 \%$ of TERT (the catalytic subunit of telomerase) is transported from the nucleus into the mitochondria, ultimately resulting in a dramatic acceleration of telomere shortening. When the cellular conditions are shifted from hyperoxia back to normoxia, TERT is re-transported back to the nucleus, and telomere length is restored (Santos et al. 2006; Ahmed et al. 2008; Saretzki 2009). Telomerase may also exert a protective effect on mitochondrial functions. Under oxidative stress, it binds to mitochondrial DNA, increases respiratory chain activity, and protects against oxidative stress-induced damage (Haendeler et al. 2009). Finally, different tissues of the bank vole Myodes glareolus from the Chernobyl Exclusion Zone displayed reduced telomere length but upregulated telomerase activity. The upregulation of telomerase, in this case, appears to be associated with functions other than telomere maintenance, perhaps protection against a stressful environment (Kesäniemi et al. 2019).

\section{Stress as a key player in telomere homeostasis maintenance}

Although organisms have evolved a set of stress responses to protect against adverse environmental conditions, protracted stressful conditions and long-term activation of the stress response negatively impact health 
and lifespan (Monaghan 2014). It is now broadly accepted that chronic stress and lifestyle factors such as oxidative stress, psychosocial stress, and improper health conditions can affect telomere dynamics (Epel et al. 2004; Kotrschal et al. 2007; Lin et al. 2012; Monaghan 2014; Korandová et al. 2018). Reproduction is an energetically costly activity that increases metabolic rates, ROS production, and susceptibility to oxidative stress, and it is hypothesized that oxidative stress may represent a mechanistic link for the inverse relationship between reproduction and lifespan in both vertebrate and invertebrate models that acts independently of energy allocation (Alonso-Alvarez et al. 2004; Wiersma et al. 2004; Krǔček et al. 2015; Sharick et al. 2015; Colominas-Ciuró et al. 2017; Costantini 2018). It has been demonstrated that resistance to oxidative stress is decreased during reproduction and that breeding activity increases susceptibility to oxidative stress (AlonsoAlvarez et al. 2004; Wiersma et al. 2004). Additionally, resistance to oxidative stress plays a significant role in shaping fecundity, as a higher fecundity rate was observed in individuals with higher oxidative protection (Bize et al. 2008). It is well established that oxidative stress in humans is implicated in pathological processes in the reproductive tract that contribute to infertility and poor pregnancy outcomes, and treatments based on strategies to boost the exhausted antioxidant defense of the reproductive microenvironment have been suggested (Adeoye et al. 2018).

DNA molecules represent an important target of oxidative damage in cells, and the most common DNA damage caused by free oxygen radicals is oxidative modifications of DNA bases such as the formation of 8-oxoguanin. Oxidative DNA lesions are associated with the production of single-strand breaks that are induced directly or as an intermediate step in the repair of oxidative base modifications. Due to their high content of guanine, telomeres are highly sensitive to oxidative damage and induction of single-strand breaks that interfere with the replication fork and thus lead to telomere attrition (von Zglinicki 2002; Houben et al. 2007; Coluzzi et al. 2019). In agreement with the assumption that breeding individuals are more susceptible to oxidative damage, engaging organisms in reproduction accelerates telomere loss (Kotrschal et al. 2007; Heidinger et al. 2011).

Bypassing the common negative association between fecundity and longevity in eusocial reproductives may explain their enhanced oxidative protection coupled with the fecundity rate. As demonstrated in ants, young founding queens exhibit immunity and resistance against environmental and physiological stress, while established older queens exhibit stimulated oxidative protection (Lucas and Keller 2018; Negroni et al. 2019). Similarly, data suggesting a role for antioxidant genes in modulating the lifespan of long-lived reproductives were identified in Adélie penguins that exhibit an increased antioxidant defense in response to breeding efforts, while oxidative damage and telomere length remained unchanged (Beaulieu et al. 2011). When we compared a few-day-old termite reproductives (with no or very low mating experience) to greater than 1year-old reproductives, the old individuals exhibited highly upregulated oxidative defenses in their somatic tissues in combination with telomerase activity (Koubová et al. 2021a; our unpublished data).

\section{What is the real purpose of telomerase activity in somatic cells ?}

To sum up, telomere shortening and lack of telomerase in most human somatic cells result in an irreversible cell cycle arrest termed replicative senescence, which is one of determinants of organismal development, an anticancer mechanism, and a marker of organismal aging. The presence of telomerase activity and telomere restoration in certain somatic cells of human adults maintains the proliferative capacity of these cells and contributes to their regenerative potential, and telomerase activity and telomere length in these cells both are commonly considered lifespan predictors (Blasco 2007; Tan et al. 2012).

However, from a broad perspective, the connection between the length of telomeres and the predicted lifespan or the reason of telomerase activity in somatic tissues of numerous animal species are not clear, which has been documented by series studies reviewed in (Gomes et al. 2010; Smith et al. 2021). For example, telomerase activity and telomere length have been studied in sea urchins in the context of the long-livedStrongylocentrotus franciscanus (live over 100 years) and the short-lived Lytechinus variegatus (with an estimated lifespan of only 3-4 years). While both species exhibit telomerase activity in their somatic tissues, L. variegatus possessed longer telomeres (average length of approximately $21 \mathrm{~kb}$ ) compared to those of $S$. franciscanus (average telomere length of approximately $5.5 \mathrm{~kb}$ ). Moreover, no differences in telomere length were observed bet- 
ween the young and old individuals. This suggests that sea urchins do not utilize telomerase repression as a mechanism to suppress neoplastic transformation (as a decline of telomerase is hypothesized to prevent tumor development), and the continuous telomerase activity in somatic tissues of the sea urchins is explained by the undetermined growth of these organisms throughout their lifespan (Francis et al. 2006). Similarly, the presence of telomerase activity throughout the lifespan or a lack of a positive correlation between the predicted lifespan, telomerase activity, and telomere length have been reported in numerous other invertebrate and vertebrate species (Gomes et al. 2010). Studies have provided no detailed information relating telomerase activity to the proliferation status of the tissues, and the presence of telomerase in the tissues was hypothetically explained by the existence of telomerase function that differs from that involved in the elongation of telomeres (Lau et al. 2008; Gomes et al. 2010).

Also, the existence of different patterns of telomerase regulation has been documented in mammals. While adult humans express telomerase almost exclusively in the germline and a few specialized cell types and possess short telomeres $(<20 \mathrm{~kb})$, most rodents express telomerase in both germline and a wide array of somatic tissues and possess long telomeres (25-150 kb) (Gomes et al. 2010). It indicates that rodents do not use telomere length to control their replicative aging. A similar lack of telomere-based replicative aging is predicted in lagomorphs (rabbits, pikas, and hares) as prolonged tissue cultures derived from different lagomorph species displayed no growth arrest or a decrease in doubling time but show either very long telomere arrays or detectable telomere activity (Forsyth et al. 2005). A comparative study examining telomerase activity and telomere length in mammals revealed that telomere length inversely correlates with lifespan (longer telomeres are present in short-lived mammal species), and telomerase activity in somatic cells inversely correlates with body mass (small mammal species such as small rodents exhibit telomerase activity in somatic cells) (Seluanov et al. 2007; Gomes et al. 2010). It has been suggested that larger and longer-lived mammals protectively repress telomerase activity in somatic cells, as their large body masses and longer lifespans contribute to an increased risk of cancer development (Seluanov et al. 2007).

\section{A role of telomerase to eliminate the cost of reproduction depending on the type of reproduc- tion strategy?}

Although telomerase has been widely demonstrated to be present in somatic tissues of numerous animal species, no detailed information is available relating telomerase to the proliferation status of the tested tissues or other biological traits that could better identify the purpose of telomerase activity in such cases. The reason for telomerase activity in non-proliferative cells remains unclear. It is speculated that in the somatic tissues of large mammals, telomerase activity is reduced as protection against tumor development, and in small mammals, telomerase is widely active, as there is no need to repress it. This "purposiveness without purpose' can be questioned in regard to why it is worthy for cells to express telomerase (or anything else) if there is no need for it. It is widely known that body mass in terrestrial mammals is negatively correlated with the fecundity rate of the species (Allainé et al. 1987; Werner and Griebeler 2011). Therefore, it is tempting to ask whether somatic telomerase activity in eusocial kings and queens as well as in small but highly fecund mammal species such as lagomorphs or rodents may serve its non-canonical function to protect its individuals against reactive oxygen species produced due to the exacerbated metabolic stress upon reproduction and may only be a reflection of a more common and broader phenomenon. We can speculate whether the necessity to maintain telomerase activity in somatic cells of some species reflects a different reproduction strategy of the species rather than their body size.

Conflicts of Interest Statement Author declares no conflict of interest.

Acknowledgements This study was supported by grant no. 18-21200S from the Grant Agency of the Czech Republic and by Strategy AV21, Diversity of Life and Health of Ecosystems.

\section{Data availability statement}

Data sharing not applicable to this article as no data sets were generated or analyzed during the current study. 


\section{References}

Aamodt R.M. (2009) Age-and caste-dependent decrease in expression of genes maintaining DNA and RNA quality and mitochondrial integrity in the honeybee wing muscle. Exp Gerontol 44:586-593. doi: 10.1016/j.exger.2009.06.004

Adeoye O., Olawumi J., Opeyemi A., Christiania O. (2018) Review on the role of glutathione on oxidative stress and infertility. J Bras Reprod Assist 22:61-66. doi: 10.5935/1518-0557.20180003

Ahmed S., Passos J.F., Birket M.J., et al. (2008) Telomerase does not counteract telomere shortening but protects mitochondrial function under oxidative stress. J Cell Sci 121:1046-1053. doi: 10.1242/jcs.019372

Allainé D., Pontier D., Gaillard J.M., et al. (1987) The relationship between fecundity and adult body weight in Homeotherms. Oecologia 73:478-480. doi: 10.1007/BF00385268

Alonso-Alvarez C., Bertrand S., Devevey G., et al. (2004) Increased susceptibility to oxidative stress as a proximate cost of reproduction. Ecol Lett 7:363-368. doi: 10.1111/j.1461-0248.2004.00594.x

Antikainen H., Driscoll M., Haspel G., Dobrowolski R. (2017) TOR-mediated regulation of metabolism in aging. Aging Cell 16:1219-1233. doi: 10.1111/acel.12689

Beaulieu M., Reichert S., Maho Y. L., et al. (2011) Oxidative status and telomere length in a long facing a costly reproductive event. Funct Ecol 25:577-585. doi: 10.1111/j.1365-2435.2010.01825.x

Ben-Avraham D., Muzumdar R.H., Atzmon G. (2012) Epigenetic genome-wide association methylation in aging and longevity. Epigenomics 4:503-509. doi: 10.2217/epi.12.41.Epigenetic

Bize P., Devevey G., Monaghan P., et al. (2008) Fecundity and survival in relation to resistance to oxidative stress in a free-living bird. Ecology 89:2584-2593. doi: 10.1890/07-1135.1

Blackburn E.H. (1991) Structure and function of telomeres. Nature. doi: 10.1038/350569a0

Blackburn E.H. (2005) Telomeres and telomerase: Their mechanisms of action and the effects of altering their functions. FEBS Lett 579:859-862. doi: 10.1016/j.febslet.2004.11.036

Blagosklonny M.V. (2010) Revisiting the antagonistic pleiotropy theory of aging: TOR-driven program and quasi-program. Cell Cycle 9:3171-3176. doi: 10.4161/cc.9.16.13120

Blasco M.A. (2007) Telomere length, stem cells and aging. Nat Chem Biol 3:640-649. doi: 10.1038/nchembio. 2007.38

Capkova Frydrychova R., Mason J.M., Biessmann H. (2009) Regulation of telomere length in Drosophila. Cytogenet Genome Res 122:356-364.

Chittka A., Chittka L. (2010) Epigenetics of royalty. PLoS Biol 8:8-11. doi: 10.1371/journal.pbio.1000532

Choudhary B., Karande A.K., Raghavan S.C. (2012) Telomere and telomerase in stem cells: relevance in ageing and disease Bibha Choudhary, Anjali A. Karande, Sathees C. Raghavan. Front Biosci 16-30.

Colominas-Ciuró R., Santos M., Coria N., Barbosa A. (2017) Reproductive effort affects oxidative status and stress in an Antarctic penguin species: An experimental study. PLoS One 12:1-15. doi: 10.1371/journal.pone. 0177124

Coluzzi E., Leone S., Sgura A. (2019) Oxidative Stress Induces Telomere Dysfunction and Senescence by Replication Fork Arrest. Cells 8:19. doi: 10.3390/cells8010019

Corona M., Hughes K.A., Weaver D.B., Robinson G.E. (2005) Gene expression patterns associated with queen honey bee longevity. Mech Ageing Dev 126:1230-1238. doi: 10.1016/j.mad.2005.07.004

Costantini D. (2018) Meta-analysis reveals that reproductive strategies are associated with sexual differences in oxidative balance across vertebrates. Curr Zool 64:1-11. doi: 10.1093/cz/zox002 
Cui H., Kong Y., Zhang H. (2012) Oxidative stress, mitochondrial dysfunction, and aging. J Signal Transduct 2012:646354. doi: $10.1155 / 2012 / 646354$

Davidovic M., Sevo G., Svorcan P., et al. (2010) Old age as a privilege of the "selfish ones". Aging Dis $1: 139-46$.

Duffy J.E., Morrison C.L., Rios .R (2000) Multiple Origins of Eusociality among Sponge-Dwelling Shrimps (Synalpheus). Evolution (N Y) 54:503-516.

Engel M.S. (2012) Monophyly and extensive extinction of advanced eusocial bees: Insights from an unexpected Eocene diversity. Proc Natl Acad Sci 98:1661-1664. doi: 10.1073/pnas.98.4.1661

Engels W. (1990) Social Insects: An Evolutionary Approach to Castes and Reproduction. Springer-Verlag, New York

Epel E.S., Blackburn E.H., Lin J., et al. (2004) Accelerated telomere shortening in response to life stress. Proc Natl Acad Sci U S A 101:17312-5. doi: 10.1073/pnas.0407162101

Fabian D., Flatt T. (2014) Life History Evolution. Nat Educ Knowl 3:1-13.

Fathi E., Charoudeh H.N., Sanaat Z, Farahzadi R (2019) Telomere shortening as a hallmark of stem cell senescence. Stem Cell Investig. doi: 10.21037/sci.2019.02.04

Forsyth N.R., Elder F.F.B., Shay J.W., Wright W.E. (2005) Lagomorphs (rabbits, pikas and hares) do not use telomere-directed replicative aging in vitro. Mech Ageing Dev 126:685-691. doi: 10.1016/j.mad.2005.01.003

Francis N., Gregg T., Owen R., et al. (2006) Lack of age-associated telomere shortening in long- and shortlived species of sea urchins. FEBS Lett 580:4713-4717. doi: 10.1016/j.febslet.2006.07.049

Gomes N.M.V., Shay J.W,. Wright W.E. (2010) Telomere Biology in Metazoa. Fed Eur Biochem Soc 584:3741-3751. doi: 10.1016/j.febslet.2010.07.031.

Greenberg R.A. (2005) Telomeres, Crisis and Cancer. Curr Mol Med 5:213-218. doi: $10.2174 / 1566524053586590$

Guo N., Parry E.M., Li L.S., et al. (2011) Short telomeres compromise $\beta$-cell signaling and survival. PLoS One. doi: 10.1371/journal.pone.0017858

Haendeler J., Dröse S., Büchner N., et al. (2009) Mitochondrial telomerase reverse transcriptase binds to and protects mitochondrial DNA and function from damage. Arterioscler Thromb Vasc Biol 29:929-935. doi: 10.1161/ATVBAHA.109.185546

Harman D. (1956) Aging: a theory based on free radical and radiation chemistry. J Gerontol 11:298 — 300. doi: 10.1093/geronj/11.3.298

Harman D. (1972) The biological clock: the mitochondria? J Am Geriatr Soc 20:99-117.

Harshman L.G., Zera A.J. (2006) The cost of reproduction : the devil in the details. Trends Ecol Evol 22:80-86. doi: $10.1016 /$ j.tree.2006.10.008

He X.J., Zhou L. B., Pan Q.Z., et al. (2017) Making a queen: an epigenetic analysis of the robustness of the honeybee (Apis mellifera) queen developmental pathway. Mol Ecol 26:1598-1607. doi: 10.1111/mec.13990

Heidinger B.J., Blount J.D., Boner W., et al. (2011) Telomere length in early life predicts lifespan. PNAS 109:1-6. doi: 10.1073/pnas.1113306109

Herb B.R., Wolschin F., Hansen K.D., et al. (2012) Reversible switching between epigenetic states in honeybee behavioral subcastes. Nat Neurosci 15:1371-1373. doi: 10.1038/nn.3218.Reversible

Hiyama E., Hiyama K. (2007) Telomere and telomerase in stem cells. Br J Cancer 96:1020-1024. doi: $10.1038 /$ sj.bjc. 6603671 
Holt S.E., Aisner D.L., Shay J.W., Wright W.E. (1997) Lack of cell cycle regulation of telomerase activity in human cells. Proc Natl Acad Sci U S A 94:10687-10692. doi: 10.1073/pnas.94.20.10687

Houben J.M.J., Moonen H.J.J., van Schooten F.J,. Hageman G.J. (2007) Chronic oxidative stress and telomere shortening. Chem Biol Interact. doi: 10.1016/j.cbi.2007.06.013

Jemielity S., Chapuisat M., Parker J.D., Keller L. (2005) Long live the queen : studying aging in social insects. Age (Omaha) 27:241-248. doi: 10.1007/s11357-005-2916-z

Jiang H., Ju Z., Rudolph K.L. (2007) Telomere shortening and ageing. Z Gerontol Geriatr 40:314-324. doi: 10.1007/s00391-007-0480-0

Jin K. (2010) Modern Biological Theories of Aging. Aging Dis 1:72-74. doi: 10.1016/j.bbi.2008.05.010

Kaiwen J., Chunmei C., Yuanxu G., et al. (2018) An analysis of aging-related genes derived from the Genotype-Tissue Expression project ( GTEx ). Cell Death Discov 4:1-14. doi: 10.1038/s41420-018-0093-y

Keller L. (1998) Queen lifespan and colony characteristics in ants and termites. Insectes soc 45:235-246.

Keller L., Genoud M (1997) Extraordinary lifespans in ants: a test of evolutionary theories of ageing. Lett to Nat 389:3-5.

Kesaniemi J., Lavrinienko A., Tukalenko E., et al. (2019) Exposure to environmental radionuclides associates with tissue-specific impacts on telomerase expression and telomere length. Sci Rep 9:1-9. doi: 10.1038/s41598-018-37164-8

Kocher S., Paxton R. (2014) Comparative methods offer powerful insights into social evolution in bees To. Apidologie 45:289-305. doi: 10.1007/s13592-014-0268-3

Korandova M., Čapková Frydrychová R. (2016) Activity of telomerase and telomeric length in Apis mellifera. Chromosoma 125:405-411. doi: 10.1007/s00412-015-0547-4

Korandová M., Krůček T., Szakosová K., et al. (2018) Chronic low-dose pro-oxidant treatment stimulates transcriptional activity of telomeric retroelements and increases telomere length in Drosophila. J Insect Physiol 104:1-8. doi: 10.1016/j.jinsphys.2017.11.002

Korandová M., Krůček T., Vrbová K., Čapková Frydrychová R. (2014) Distribution of TTAGG-specific telomerase activity in insects. Chromosom Res 22:495-503.

Kotrschal A.., Ilmonen P, Penn D.J. (2007) Stress impacts telomere dynamics. Biol Lett 3:128-130. doi: 10.1098/rsbl.2006.0594

Koubová J., Čapková Frydrychová R. (2021) Telomerase-positive somatic tissues of honeybee queens (Apis mellifera) display no DNA replication. Cytogenet Genome Res Oct 14:1-6.

Koubová J., Jehlík T., Kodrik D., et al. (2019) Telomerase activity is upregulated in the fat bodies of pre-diapause bumblebee queens (Bombus terrestris). Insect Biochem Mol Biol 115:103241.

Koubová J., Pangrácová M., Jankásek M., et al. (2021a) Long-lived termite kings and queens activate telomerase in somatic organs. Proc R Soc B 288:20210511.

Koubová J., Sábová M., Brejcha M., et al. (2021b) Seasonality in telomerase activity in relation to cell size , DNA replication, and nutrients in the fat body of Apis mellifera. Sci Rep 11:1-11.

Krůček T., Korandová M., Šerý M., et al. (2015) Effect of low doses of herbicide paraquat on antioxidant defense in Drosophila. Arch Insect Biochem Physiol 88:235-48. doi: 10.1002/arch.21222

Kurz D.J., Decary S., Hong Y., et al. (2004) Chronic oxidative stress compromises telomere integrity and accelerates the onset of senescence in human endothelial cells. J Cell Sci 117:2417-26. doi: 10.1242/jcs.01097 
Lau B.W.M., Wong A.O.L., Tsao G.S.W., et al. (2008) Molecular cloning and characterization of the zebrafish (Danio rerio) telomerase catalytic subunit (telomerase reverse transcriptase, TERT). J Mol Neurosci 34:6375. doi: 10.1007/s12031-007-0072-x

Laurent K., Genoud M. (1997) Extraordinary lifespans in ants: a test of evolutionary theories of ageing. Nature 389:958-960.

Lin J., Epel E, Blackburn E. (2012) Telomeres and lifestyle factors: roles in cellular aging. Mutat Res 730:859. doi: $10.1016 /$ j.mrfmmm.2011.08.003

Liu L., Trimarchi J.R., Smith P.J.S., Keefe D.L. (2002) Mitochondrial dysfunction leads to telomere attrition and genomic instability. Aging Cell 1:40-46. doi: 10.1046/j.1474-9728.2002.00004.x

Lucas E.R., Keller L. (2018) Elevated expression of ageing and immunity genes in queens of the black garden ant. Exp Gerontol 108:92-98. doi: 10.1016/j.exger.2018.03.020

Lyko F., Foret S., Kucharski R., et al. (2010) The Honey Bee Epigenomes : Differential Methylation of Brain DNA in Queens and Workers. doi: 10.1371/journal.pbio.1000506

Mason J.M., Randall T., Capkova Frydrychova R. (2015) Telomerase lost? Chromosoma. doi: 10.1007/s00412-015-0528-7

Mason J.M., Reddy H.M., Capkova Frydrychova R (2011) Telomere maintenance in organisms without telomerase. In: Seligman H (ed) DNA Replication-Current Adv. InTech, pp 323-346

Monaghan P. (2014) Organismal stress, telomeres and life histories. J Exp Biol 217:57-66. doi: $10.1242 /$ jeb.090043

Murphy M.P. (2009) How mitochondria produce reactive oxygen species. Biochem J 417:1-13. doi: 10.1042/BJ20081386

Negroni M.A., Foitzik S., Feldmeyer B. (2019) Long-lived Temnothorax ant queens switch from investment in immunity to antioxidant production with age. Sci Rep 9:1-10. doi: 10.1038/s41598-019-43796-1

Ozawa T. (1997) Genetic and functional changes in mitochondria associated with aging. Physiol Rev 77:425464 .

Passos F., Saretzki G., Ahmed S., et al. (2007) Mitochondrial Dysfunction Accounts for the Stochastic Heterogeneity in Telomere-Dependent Senescence. Plos Biol 5:1138-1151. doi: 10.1371/journal.pbio.0050110

Razgonova M.P., Zakharenko A.M., Golokhvast K.S., et al. (2020) Telomerase and telomeres in aging theory and chronographic aging theory ( Review ). Mol Med Rep 22:1679-1694. doi: 10.3892/mmr.2020.11274

Reznick D. (1985) Costs of reproduction: an evaluation of the empirical evidence. Oikos 44:257-267.

Sahin E., Colla S., Liesa M., et al. (2011) Telomere dysfunction induces metabolic and mitochondrial compromise. Nature 470:359-365. doi: 10.1038/nature09787. Telomere

Santos J.H., Meyer J.N., Van Houten B. (2006) Mitochondrial localization of telomerase as a determinant for hydrogen peroxide-induced mitochondrial DNA damage and apoptosis. Hum Mol Genet 15:1757-1768. doi: $10.1093 / \mathrm{hmg} / \mathrm{ddl} 098$

Saretzki G. (2009) Telomerase, mitochondria and oxidative stress. Exp Gerontol 44:485-92. doi: 10.1016/j.exger.2009.05.004

Segal-Bendirdjian E., Geli V., Cayuela M.L. (2019) Non-canonical Roles of Telomerase : Unraveling the Imbroglio. Front Cell Dev Biol 7:1-12. doi: 10.3389/fcell.2019.00332

Seluanov A., Chen Z., Hine C., et al. (2007) Telomerase activity coevolves with body mass, not lifespan. Aging Cell 6:45-52. doi: 10.1111/j.1474-9726.2006.00262.x. Telomerase 
Sharick J.T., Vazquez-Medina J.P., Ortiz R.M., Crocker D.E. (2015) Oxidative stress is a potential cost of breeding in male and female northern elephant seals. Funct Ecol 29:367-376. doi: 10.1111/1365-2435.12330

Sherman P.W., Lacey E.A., Reeve H.K., Keller L. (1994) The eusociality continuum. Behav Ecol 6:102-108.

Smith S., Hoelzl F., Zahn S., Criscuolo F. (2021) Telomerase activity in ecological studies: what are its consequences for individual physiology and is there evidence for effects and trade-offs in wild populations. Mol Ecol 1-13. doi: 10.1111/mec.16233

Tan T.C.J., Rahman R., Jaber-Hijazi F., et al. (2012) Telomere maintenance and telomerase activity are differentially regulated in asexual and sexual worms. Proc Natl Acad Sci U S A 109:4209-14. doi: $10.1073 /$ pnas. 1118885109

Ulaner G.A., Giudice L.C. (1997) Developmental regulation of telomerase activity in human fetal tissues during gestation. Mol Hum Reprod 3:769-773.

Werner J., Griebeler E.M. (2011) Reproductive biology and its impact on body size: Comparative analysis of mammalian, avian and dinosaurian reproduction. PLoS One. doi: 10.1371/journal.pone.0028442

Wiersma P., Selman C., Speakman J.R., Verhulst S. (2004) Birds sacrifice oxidative protection for reproduction. Proc R Soc B Biol Sci 271:360-363. doi: 10.1098/rsbl.2004.0171

Wilson E.O. (1971) The Insect Societies. Cambridge Belknap Press of Cambridge University Press. 1:548.

Wright W.E., Piatyszek M.A., Rainey W.E., et al. (1996) Telomerase activity in human germline and embryonic tissues and cells. Dev Genet 18:173-179.

von Zglinicki T. (2002) Oxidative stress shortens telomeres. Trends Biochem Sci 27:339-44.

Zheng Q., Huang J., Wang G., et al. (2019) Mitochondria, Telomeres and Telomerase Subunits. Front Cell Dev Biol 7:1-10. doi: 10.3389/fcell.2019.00274

Zhu X., Kumar R., Mandal M., et al. (1996) Cell cycle-dependent modulation of telomerase activity in tumor cells. PNAS 93:6091-6095. 